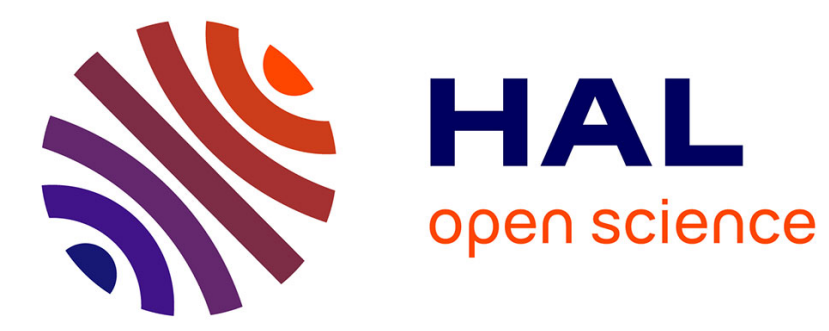

\title{
Current sharing between parallel turns of a planar transformer: prediction and improvement using a circuit simulation software
}

Xavier Margueron, Jean-Pierre Keradec, Abdelhadi Besri

\section{To cite this version:}

Xavier Margueron, Jean-Pierre Keradec, Abdelhadi Besri. Current sharing between parallel turns of a planar transformer: prediction and improvement using a circuit simulation software. IEEE Industrial Application Society 2007, Sep 2007, New Orléans, United States. hal-00289169

\section{HAL Id: hal-00289169 \\ https://hal.science/hal-00289169}

Submitted on 13 Feb 2009

HAL is a multi-disciplinary open access archive for the deposit and dissemination of scientific research documents, whether they are published or not. The documents may come from teaching and research institutions in France or abroad, or from public or private research centers.
L'archive ouverte pluridisciplinaire HAL, est destinée au dépôt et à la diffusion de documents scientifiques de niveau recherche, publiés ou non, émanant des établissements d'enseignement et de recherche français ou étrangers, des laboratoires publics ou privés. 


\title{
Current Sharing Between Parallel Turns of a Planar Transformer: Prediction and Improvement Using a Circuit Simulation Software
}

\author{
X. Margueron, J-P. Keradec, A. Besri \\ G2Elab \\ UMR 5269-INPG-UJF-CNRS \\ ENSIEG, B.P. 46, 38402 St Martin d'Hères Cedex, France \\ Jean-Pierre.Keradec@g2elab.inpg.fr
}

\begin{abstract}
With the increase of power needs in low voltage applications, wiring several windings in parallel to sustain large currents becomes common. This operation often has a dangerous impact on transformer reliability because additional currents, we call "circulation currents", create extra losses, generally not taken into account by analytical approaches. Yet, hot points which result from these currents can destroy the component. In planar transformers, windings are made of PCB layers and circulation currents lead to severe unbalance of current sharing between parallel layers. This paper presents an analytical method which enables currents in all layers to be evaluated using only a circuit simulation software such as Pspice ${ }^{\circledR}$ or PSIM $^{\circledR}$. For a designer, this method is very intuitive and fast compared with the use of fem simulations.
\end{abstract}

Keywords-planar transformers; PCB parallel windings; circulation current; model; analytical calculation.

\section{INTRODUCTION}

Frequency rise in power electronic converters has induced a diminution of active and passive component sizes. Above $100 \mathrm{kHz}$, traditional round winding transformers are often replaced by planar ones which use low profile magnetic cores and printed circuit board (PCB) for turns.

For actual applications which need high output currents, the use of PCB conductors 50-150 $\mu \mathrm{m}$ thick is problematic. Even if it is some $\mathrm{cm}$ wide, cross section of one layer remains small so several layers have to be wired in parallel to sustain several hundredths of Amps [1]. Unfortunately, paralleling several layers doesn't result automatically in a balanced current sharing. Indeed, flux flowing through these parallel turns is slightly different and so are induced voltages. As a result, additional currents, here called "circulation currents", are created by these low differential voltages. These internal extra currents are only limited by turn impedances so they seriously impact component losses and reliability. Consequently, accounting for them is not optional for the designer.

At first sight, to design a transformer and predict the current distribution between parallel layers, fem simulations can be used [2]. In practice, planar transformers are quite complex-we studied one including 44 conductive layers-so detailed description of shape and physical properties is time consuming. Worst, to correctly account for eddy currents, meshing must be fine enough so a very large memory computer is needed to carry out a simulation at around $1 \mathrm{MHz}$ and such a simulation lasts several hours.

In order to short the "engineer-time" needed to study such a planar transformer intended for an industrial application and to ease optimization process, we developed a method, based on an analytical approach, which enables the designer to obtain the needed results by using any circuit simulation software [3]. Such software is among the familiar tools of the designer and related simulations last seconds rather hours, even for complex multi-layer planar transformer.

\section{PROBlem Due to PARALELl Windings}

Impact of eddy currents on copper losses of a high frequency has been widely studied. Several analytical methods have been proposed to account for these effects, especially when windings can be represented as stacks of conductive layers insulated from each other $[4,5]$. They all start from the solution of propagation equation in an infinite plan layer made of conductive linear homogenous and isotropic material. For one layer, total losses split into skin and proximity losses.

In a transformer, when wires are connected in parallel, adding skin and proximity losses of all wires leads to total copper losses only if currents flowing in all wires connected in parallel have not been supposed equal from the beginning. Indeed, as explained above, circulation currents add to the mean current per wire so skin losses must be calculated with the real current that flows in it.

Recently we presented the effect of circulating currents into a $9 \mathrm{kVA}, 125-\mathrm{kHz}$, planar transformer (Fig. 1) [6]. This industrial transformer includes 44 conductive layers. Its PQ core [7] is made of ferrite PC40. This component has two secondaries, each made of 11 paralleled layers. Its primary is made of 11 pairs of layers. Each pair is wired in parallel, then, all these pairs are connected in series. Due to external circuit, while one secondary is loaded (output current : $275 \mathrm{~A}_{\text {eff }}$ ), the other is in open circuit. The loaded is modeled by a $0.12 \Omega$ resistance. 


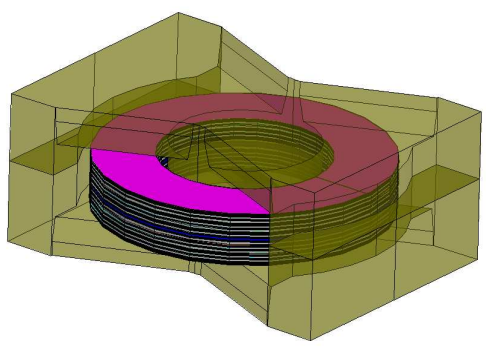

Figure 1. 44-conductive layers, 3 windings planar transformer

Eleven primary layers are located above and eleven under the secondaries. The current calculated in the different layers, as it is obtained with $2 \mathrm{D}$ fem. simulation software [8], is presented in Fig. 2.

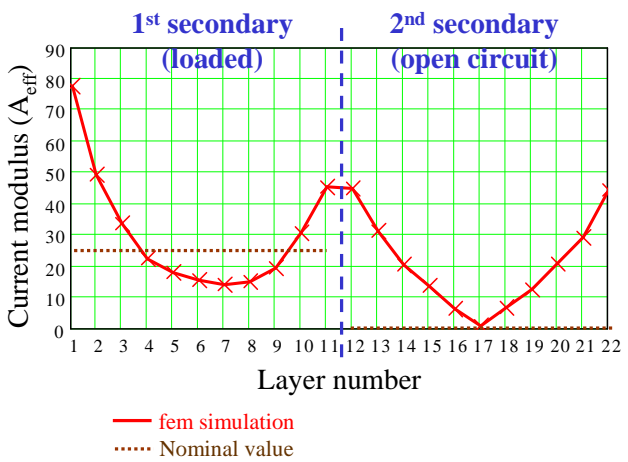

Figure 2. Current repartition in secondary layers. Current modulus is plotted versus secondary layer number

For the loaded secondary, nominal current should be of 25 $A_{\text {eff }}$ per layer. The maximal current value $\left(80 \mathrm{~A}_{\text {eff }}\right)$, which appears for the external layer of the loaded winding, is more than three times higher so, power losses in this layer is ten times than expected. For the open circuit winding, despite nominal current is null (Fig. 2 shows only modulus!), currents in layers 12 and 22 reach $40 \mathrm{~A}_{\text {eff. }}$.

Even if it doesn't impact on transformer efficiency (still above $98.5 \%$ ), this catastrophic current sharing results in strongly localized extra losses (Fig. 3), even in the open circuit secondary. That creates hot points into PCB and, sometimes, destroys the transformer.

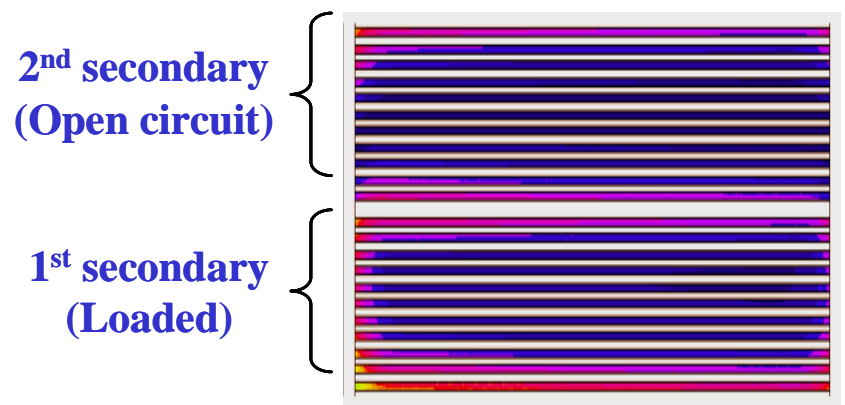

Figure 3. Power repartition in secondary layers. The Clearer the color is, the higher the power density is

\section{HOW TO PREDICT CIRCULATION CURRENTS ?}

The impact of circulating currents may be destructive for transformer. Designers have to predict them while they are dimensioning their components.

\section{A. Limits of fem Simulations}

Traditional method for determining circulation current is based on fem simulation. Introducing planar transformer geometry in software is quite easy but it is a long task. Besides, meshing of such a component (for example the 44 layers one) must place 2 mashes in a skin depth to properly account for eddy currents. As a consequence, calculation requires a large memory size computer. In practice, the major problem arises when geometrical optimizations are carried out. In order to find the best topology for loss reduction, numerous simulations have to be run $[9,10]$ and both description and computation times are often prohibitive.

In order to reduce transformer's development and optimization time, we looked for an analytical method convenient to easily and quickly check different topologies.

\section{B. Analytical Method : 1-D propagation assumption}

\section{1) Principe}

Looking at its 2D cross section, a planar transformer can be seen as a stack of different types of layers made of conducting, insulating or magnetic material (Fig. 4). Each material is considered as linear, homogeneous, isotropic and it is described by its complex permeability and its complex permittivity.

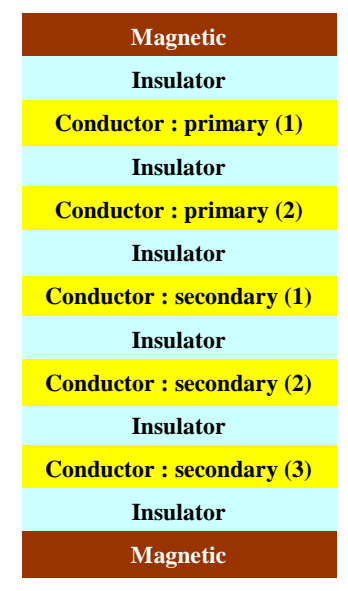

Figure 4. Example of layer stack

Assuming energy propagation is 1-D (normal to the layers) $[11,12]$, power exchange of a layer is defined, by 6 parameters linearly linked: electric and magnetic fields on each faces, and supplied current and voltage (if the layer is conductive). Such an approach leads to a 3-port equivalent circuit that synthetically represents all energy transfers of a layer.

In order to develop this unidirectional approach, let's consider an infinite plane layer of thickness $a$, perpendicular to $O z$ axis. A portion of this layer (height $b$ and depth $c$ ) is taken into account (Fig. 5). This plate is exposed to plane waves (incident and reflected) on its two faces. Magnetic field $H$ is parallel to $O y$ while electric field $E$ is directed toward 
$O x$. If this plate is a conductive one, a conduction courant can flow inside. This layer is submitted to three power flux: one at the input, one at the output and the third is injected.

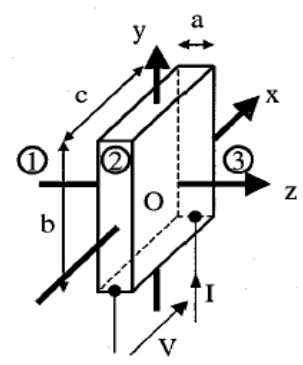

Figure 5. One layer

Relations between the six external variables $\left(E_{g}\right.$ and $H_{g}$ at the input, $E_{d}$ and $H_{d}$ at the output, $V$ and $I$ ) can be written as a $3 \times 3$ matrix which is associated to a passive 3-port circuit. However, Ampere's theorem shows that current $I$ is linked to magnetic excitation $\left(H_{g}\right.$ and $H_{d}$ ) on both faces (1).

$$
H_{g}-H_{d}=J
$$

As a consequence, matrix relation is reduced to a couple of equations (2) [11] that can be represented by a 2-port circuit (Fig. 6).

$$
\begin{gathered}
E_{g}-E_{s}=Z_{1} \cdot H_{g}+Z_{2} \cdot J \\
E_{d}-E_{s}=-Z_{1} \cdot H_{d}+Z_{2} \cdot J
\end{gathered}
$$

with $J=-\frac{I}{b}$ and $E_{s}=-\frac{V}{c} \Leftrightarrow Z=\frac{V}{I}=\frac{E_{s}}{J} \frac{c}{b}$

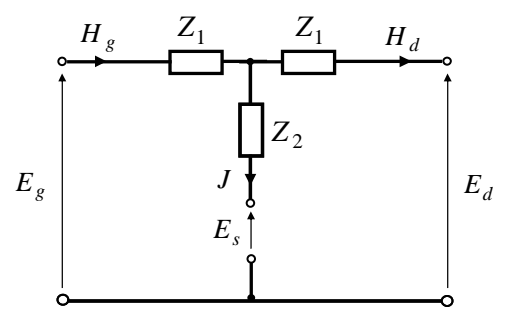

Figure 6. Equivalent 2-port circuit (quadripolar form)

Owing to relation (3), this circuit can be modified to introduce potential $V$ and current $I$ (Fig. 7)

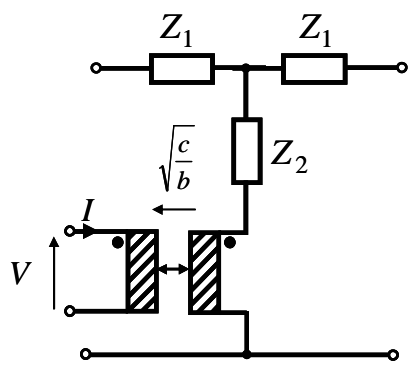

Figure 7. Equivalent circuit for a conductive layer

\section{2) Impedance formulation}

Analytical expressions of impedances $Z_{1}$ and $Z_{2}$ (Fig. 7) depend on complex phase $\phi$ (4) and on attenuation $A$ (5) introduced by the plate of thickness $a$. They depend also on characteristic impedance $Z_{c}$ of this media (6).

$$
\begin{gathered}
\phi=\omega \sqrt{\bar{\mu} \bar{\varepsilon}} \cdot a \\
A=e^{-j \phi} \\
Z_{c}=\sqrt{\frac{\bar{\mu}}{\bar{\varepsilon}}}
\end{gathered}
$$

$\mathrm{Z} 1$ and $\mathrm{Z} 2$ can then simply be written with (7) and (8).

$$
\begin{aligned}
& Z_{1}=Z_{c} \frac{1-A}{1+A}=Z_{c} \cdot j \cdot \tan \left(\frac{\phi}{2}\right) \\
& Z_{2}=Z_{c} \frac{2 A}{1-A^{2}}=Z_{c} \frac{1}{j \cdot \sin (\phi)}
\end{aligned}
$$

These formulas are general and they need to be personalized to any type of material present in transformers. For example, only conductive layers need an electrical supply. Other types of material, not supplied, are represented by a serial impedance $\left(2 Z_{1}\right)$.

To study a transformer, three different types of material need to be described: conductive, insulating and magnetic ones.

a) Conductive layer (copper...)

A conductive layer is defined by its resistivity (or its conductivity $\sigma) \rho=\frac{1}{\sigma}$ and its thickness $e p$. For a conductor, permittivity $\varepsilon$ is directly linked to conductivity $\sigma$ (9) so $\phi$ and $Z_{c}$ are expressed as (10) and (11).

$$
\varepsilon=\frac{\sigma}{j \cdot \omega}
$$

$$
\begin{aligned}
& \phi=\omega \sqrt{\frac{\sigma}{j \cdot \omega} \mu_{0}} \cdot e p=(1-j) \frac{e p}{\delta} \\
& Z_{c}=\sqrt{j \cdot \omega \cdot \rho \cdot \mu_{0}}=(1+j) \frac{1}{\sigma \delta}
\end{aligned}
$$

With $x=\frac{e p}{\delta}, Z_{1}$ and $Z_{2}$ become :

$$
Z_{1}=r_{0} \cdot x \cdot \frac{\sinh (x)-\sin (x)+j \cdot[\sinh (x)+\sin (x)]}{\cosh (x)+\cos (x)}
$$




$$
\begin{gathered}
Z_{2}=r_{0} \cdot 2 x \cdot \frac{\sinh (x) \cdot \cos (x)+\cosh (x) \cdot \sin (x)}{\cosh (2 x)-\cos (2 x)} \\
+\frac{j \cdot[\sinh (x) \cdot \cos (x)-\cosh (x) \cdot \sin (x)]}{\cosh (2 x)-\cos (2 x)} \\
\text { with } r_{0}=\frac{1}{\sigma \cdot a}
\end{gathered}
$$

b) Air layer (or insulating)

An insulating layer (thickness : $e p_{-} a$ ) is modelled by a simple inductance (14).

$$
Z_{a}=i \cdot \omega \cdot \mu_{0} \cdot e p_{-} a \Leftrightarrow \mu_{0} e p_{-} a=L
$$

\section{c) Magnetic layer (ferrite)}

A magnetic layer is characterized by its thickness $e p_{-} f$ and its permeability $\mu_{r}$. The simplest model for such a layer is an inductance (15). If necessary, losses can be taken into account by adding a parallel resistance and even more sophisticated models have been developed [13]. However, at long as we focus essentially on copper losses such sophisticated models are useless. For the same reasons, fem simulations will be carried out assuming permeability is real.

$$
Z_{f}=i \cdot \omega \cdot \mu_{0} \cdot \mu_{r} \cdot e p_{-} f \Leftrightarrow \mu_{0} \mu_{r} e p_{-} f=L
$$

\section{3) Example}

Let's take a simple two windings EP transformer as an example. Each winding is assumed to be made of only one layer. Axial symmetry of this device enables to obtain a layer stack after unrolling the transformer (Fig. 8). Depth $c$ is taken equal to average turn length. Height $b$ is equal to layer's width. Each layer is separated from the others by an insulating zone which has the same permeability than air. Turns number of primary and secondary are introduced in the equivalent circuit by the mean of coupler ratios [12] which links the layer to its power supply. Finally, the equivalent circuit obtained this way is presented on Fig. 8 .

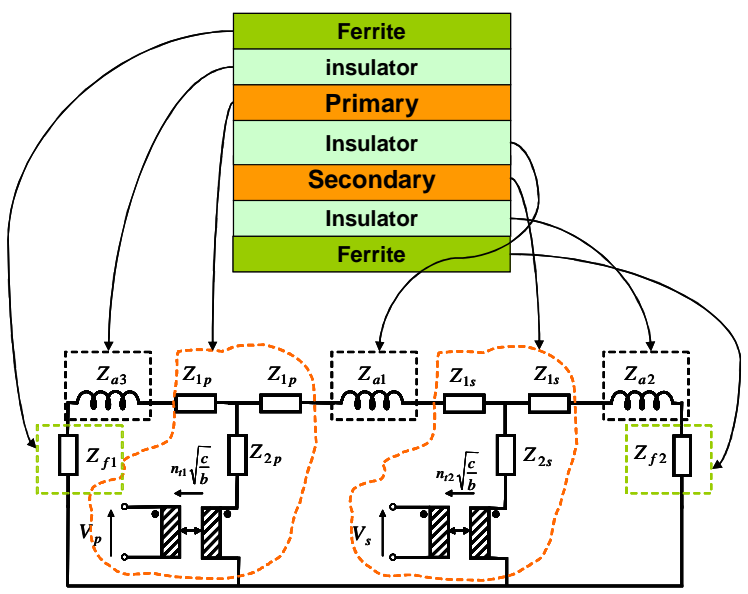

Figure 8. Equivalent circuit for a two winding transformer
After some elementary transformations, this equivalent circuit looks more familiar, as one can see on Fig. 9. All impedances depend on frequency parameter. Open circuit impedance of this transformer depends essentially of magnetic impedance $\left(\eta_{1}^{2} \cdot\left(Z_{1 p}+Z_{a 3}+Z_{f 1}\right)\right)$. In this expression, ferrite impedance $Z_{f 1}$ is dominating.

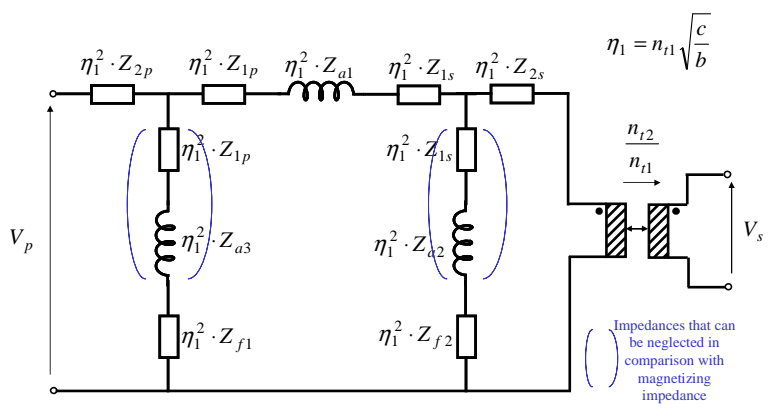

Figure 9. Classical model (similar to Fig. 8)

\section{4) Application to 44-layers transformer}

Geometry of 44-layers transformer (Fig. 3) seems very interesting to check the equivalent circuit representation introduced above. In fact, all conductors have the same width and they are separated by identical insulating layers.

In order to implement this model in circuit simulation software [3], 309 impedances (3 resistances and 3 reactances per layer) and 44 couplers must be introduced. Results obtained with this model are presented in Fig. 10.

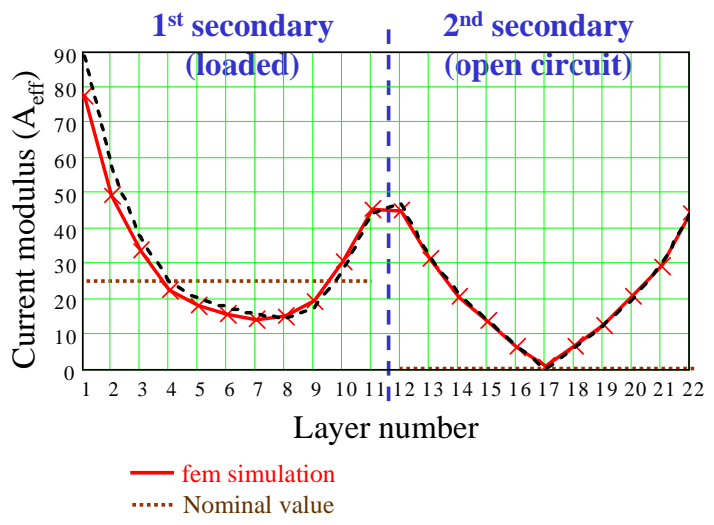

Figure 10. Comparison of current repartition in secondary layers

This simulation gives very good results. Current in the load is equal to $275 \mathrm{~A}_{\text {eff }}$ and the repartition obtained with fem software is quite similar to that deduced from our equivalent circuit representation introduced in circuit simulation software (difference less than 5\%). The most interesting aspect of this "analytical simulation" is that it is very fast. Computing time is less than $20 \mathrm{~s}$ and this can be advantageously compared to the 20 min necessary to run an fem simulation. Introduction of such model into a circuit software is also simpler (and faster) because numerous identical cells can be repeated.

In the final part of this paper, different layer stacks will be compared in order to reduce circulation currents and to improve transformer reliability. 


\section{PlanAR TRANSFORMER STUDIES}

\section{A. Reduction of circulation currents}

Circulation currents are due to leakage flux enclosed by parallel layers and these flux increase with the distance separating two conductive layers. So, best solution appears to be the use only one thick layer with the total copper thickness! In standard transformers, one alternative solution consists in twisting wires such as in Litz wires. This does reduce the flux embraced by each pair of wires but it is not applicable for PCB windings.

The second solution consists in modifying winding arrangement to reduce the leakage inductance of the transformer [14]. This is achieved by interleaving primary and secondary to locally cancel Ampere-turns. This solution, which reduces rms induction in the transformer window when one winding is shorted, decreases stored energy and leakage inductance. It also reduces eddy current losses which are roughly proportional to this rms induction.

\section{B. Layer stack improvements}

Impact of interleaving is now tested using our equivalent circuit method. All layers stay at the location occupied in the 44-layer transformer but they are connected differently. This leads to the three different layer stacks shown in Fig. 11.

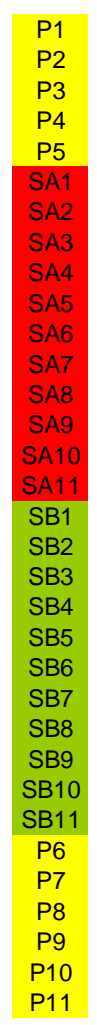

(a)

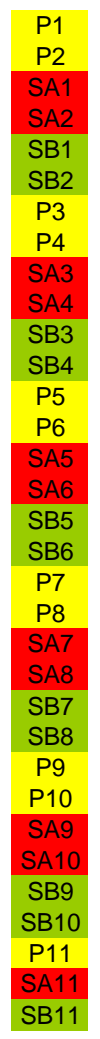

(b)

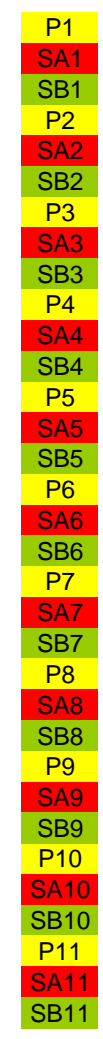

(c)
Figure 11. Connections of the 44 conductive layers of the 3 -winding transformer. (a) : initial design | (b) : arrangement $1 \mid$ (c) : arrangement 2
The first one (Fig. 11-a) describes the initial planar device. Each primary layer in the figure is composed of two parallel layers. Secondaries are both placed between the two halfprimaries. The second design (Fig. 11-b) is obtained by interleaving 2 primary layers, 2 "A" secondary layers and 2 "B" secondary layers. The third one (Fig. 11-c) consists in interleaving the three winding layers one by one. According to our guiding idea, this last arrangement seems to be the ideal one but can not always be done because of manufacturing reasons.

Comparison between theses different arrangements can be realized very quickly with the use of circuit simulation software. Only connections between plates must be modified to achieve different arrangements.

Fig. 12 presents the comparison of currents in secondary A (loaded winding). Both arrangements 1 and 2 lead to quite equal currents in parallel layers.

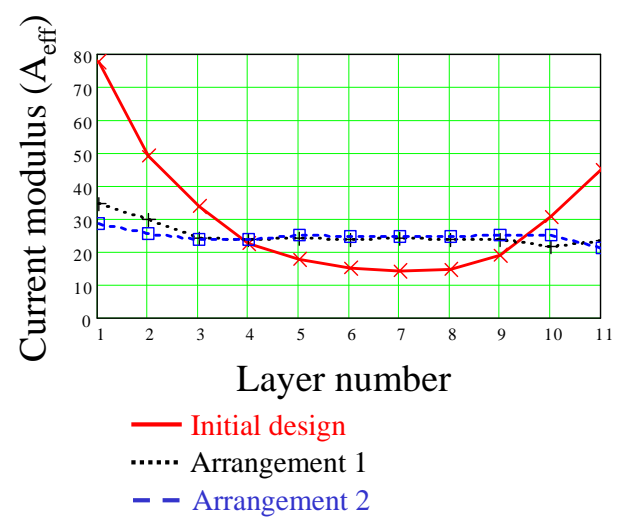

Figure 12. Current distribution in loaded winding (secondary A)

For the open circuit winding (Secondary B), currents values obtained with circuit simulation are presented on Fig. 13. Circulations currents are reduced to $5 \mathrm{~A}_{\text {eff }}$ with the arrangement 1. This value can be compare to the $45 \mathrm{~A}_{\text {eff }}$ reached in the initial device. Arrangement 2 enables to still reduce currents to $1 \mathrm{~A}_{\mathrm{eff}}$ (nominal values may be null).

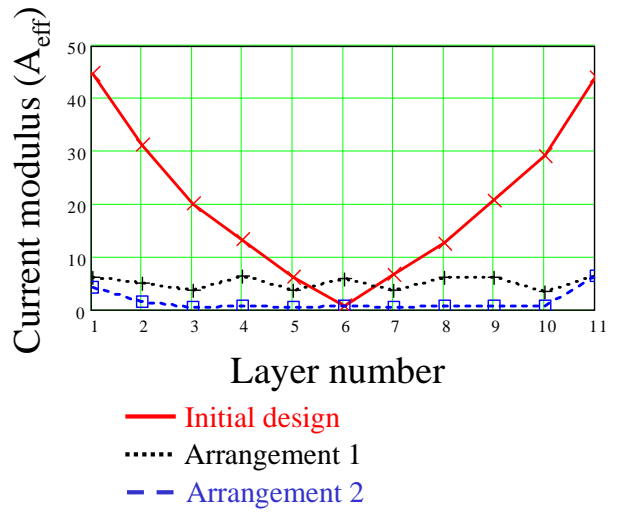

Figure 13. Current repartition in open circuit winding (secondary B)

Total power dissipated in copper can also be compared: for the initial design, it is evaluated to $70 \mathrm{~W}$, for arrangement 1 , losses are reduced to $19 \mathrm{~W}$ and, for arrangement 2 , losses still 
decrease slightly to $17.8 \mathrm{~W}$. This values doesn't include losses in the PCB vias and in the magnetic core.

\section{Final optimized transformer}

Owing to our equivalent circuit method, connection between layers as well as thickness of insulating and conductive layers can be changed easily and quickly. However, the solution showing lowest losses is not necessary the one that engineers adopt. Other industrial considerations are taken into account such as volume, weight, cost, .... The study of this 44layers planar transformer has been proposed by Thales and it gave us the opportunity of working with Thales engineers to improve their component. After balancing best efficiency solution with cost and ease of manufacturing, they built the component described below (Fig. 14).

This component is made of a smaller number of layers. Both secondary are made of 6 parallel layers. Thickness of PCB layers have been increased (surface x1.5) to sustain $275 / 6=45.8 \mathrm{~A}_{\text {eff. }}$ Arrangement of primary and both secondary is a mix ( 2 primaries- 1 secondary A-1 secondary B) between Fig. 11-b and Fig. 11-c. Final performances are presented below.

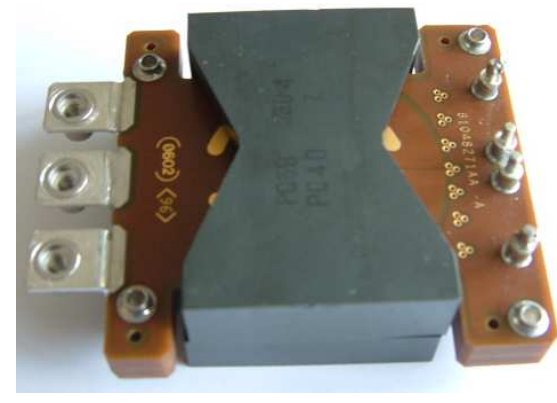

Figure 14. 24-layers optimized transformer

Currents repartitions in secondary layers are presented in Fig. 15 for the loaded secondary, and in Fig. 16 for the open circuit secondary. On these figures, results obtained with fem software are compared with those deduced using circuit simulation software. Results are in very good agreement.

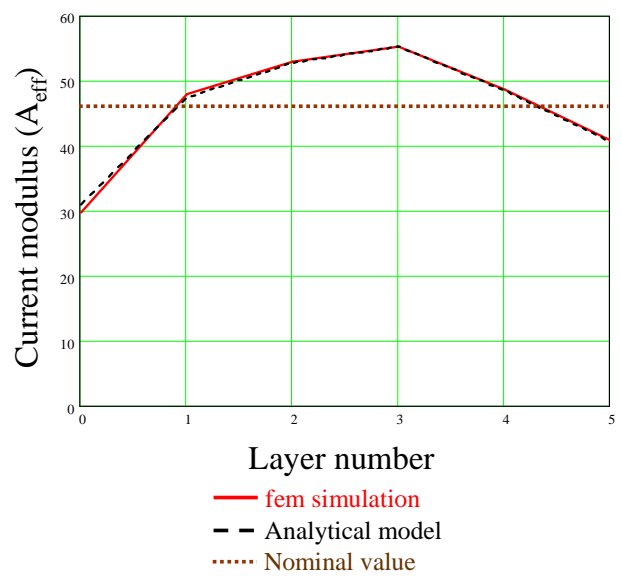

Figure 15. Currents repartition in optimized 24-layer planar transformer (Secondary A)
Current in loaded windings is included between $30 \mathrm{~A}_{\text {eff }}$ and $55 \mathrm{~A}_{\text {eff }}$. This values must be compared to the nominal value : $45.8 \mathrm{~A}_{\text {eff. }}$ In the open circuit secondary, current is included between $2 \mathrm{~A}_{\text {eff }}$ and $13 \mathrm{~A}_{\text {eff. }}$ Copper losses are close to $20.6 \mathrm{~W}$ and, according to measurements, power efficiency seems to be slightly above $99 \%$.

Compared to the first prototype, better performances and reliability are obtained (lower copper losses, reduce hot spots) and this component is easier to manufacture.

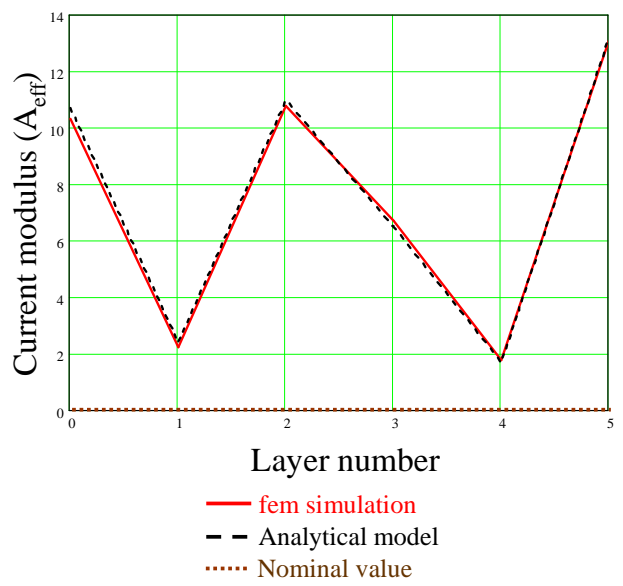

Figure 16. Currents repartition in optimized 24-layer planar transformer (Secondary B)

\section{CONCLUSION}

In this paper, we investigated the impact of circulation currents on high frequency transformers. These currents appear as soon as at least one winding is made of strands connected in parallel. They are never taken into account in analytical approaches. They can be studied by f.e.m. simulations providing equality of currents circulating in parallel strands is not imposed from the beginning.

Despite paralleling winding seems to be a good solution to reduce eddy current effects while sustaining high frequency strong currents, impact of circulation currents must be evaluated to avoid destructive hot spots. This evaluation is long and quite incompatible with an optimization process when it is done using traditional approach (analytical and f.e.m. simulation).

In this paper, an exact analytical approach has been formatted to appear as a circuit problem that can be solved using standard circuit simulation software. This method, based on the 1-D propagation assumption, leads to an equivalent circuit suitable to determine all layer currents, including circulation ones. Owing to this tool, checking concurrent topologies for a planar transformers with given specification is easy and fast. Using it, engineers can save a lot of time.

Since this paper have been proposed, equivalent circuit method has been extended and it is now usable with non harmonic waveforms. This will be published soon. 


\section{REFERENCES}

[1] C. Wei, Y. Yipen, H. Yuenquan, L. Quing, "Model and design of PCB parallel winding for planar transformer", IEEE Transactions on Magnetics, Vol. 39, No. 5, pp. 3202-3204, September 2003.

[2] X. Shangyang, Q. Jingen, W. Thomas, N. Khai, Z. Hua, "On winding design of planar transformer for miniaturized megahertz DC-DC converters", 11th International Conference on Power Electronics and Motion Control, EPE-PEMC04, Riga, Latvia.

[3] PSIM 6.0, Power Sim Inc, http://www.powersimtech.com.

[4] A. Reatti and K. Kazimierczuk, "Comparison of various methods for calculating the AC resistance of inductors", IEEE Transactions on Magnetics, Vol 38, n³, pp. 1512 - 1518, May 2002.

[5] R. Prieto, J. A. Oliver, J. A. Cobos, J. Uceda, "1D magnetic component model for planar structures", IEEE Power Electronics Specialists Conference, PESC99, June 1999, Charleston (SC), Vol. 1, pp.574-579.

[6] X. Margueron, J-P. Keradec, H. Stephan, "Les courants de circulation dans les conducteurs en parallèle : Influence dans un transformateur planar", Electronique de Puissance du Futur, EPF06, Grenoble, 5-6 juillet 2006. Proc.S8-8.

[7] www.tdk.de/productsearch/ferrite.html.

[8] Flux, Cedrat, 10 Chemin de Pré Carré-ZIRST, 38426 Meylan, France. http://www.cedrat.com
[9] R. Prieto, J. A. Cobos, O. Garcia, P. Alou, J. Uceda, "Using parallel Windings in Planar Magnetic Components", IEEE Power Electronics Specialists Conference, PESC01, June 2001, Vol. 4, pp. 2055-2060.

[10] G. R. Skutt, P. S. Venkatraman, "Analysis and measurement of highfrequency effects in high-current tranformers", Applied Power Electronics Conference and Exposition, APEC90, March 1990, pp. 354364.

[11] J-P. Keradec, B. Cogitore, F. Blache, "Power transfer in a two winding transformer: From 1D propagation to an equivalent circuit", IEEE Transactions on Magnetics, Vol. 32, No. 1, January 1996.

[12] A. Schellmanns, P. Fouassier, J-P. Keradec, J-L. Schanen, "Equivalent circuits for transformers based on one-dimensional propagation: Accouting for multilayer structure of windings and ferrite losses", IEEE Transactions on Magnetics, Vol. 35, No. 5, September 2000.

[13] J-P. Keradec, P. Fouassier, B. Cogitore, F. Blache "Accounting for Resistivity and Permeability in High Frequency Permeability measurements: Application to MnZn Ferrites", IEEE Instrumentation and Measurement Society, IMTC03, Vol. 2, May 2003, Vail USA, pp. 1252-1256.

[14] H. Yuequan, G. Junfeng, B. Xinmin, C. Wie, "Problems of paralleling windings for planar transformer and solutions", IEEE Power Electronics Specialists Conference, PESC02, June 2002, Vol. 2, pp. 597-601. 